\title{
Als Doktor Google und Doktor B. mir weiterhalfen
}

\section{Erica BrühImann-Jecklin}

Psychotherapeutin SPV / Supervisorin, Schriftstellerin und Liedermacherin

«Ich habe eben Läuse und Flöhe!», sage ich jeweils zur Erklärung meiner Geh- und Sehbehinderung. «Damit kann man leben, hat eine Perspektive."

Dann der Januar 2017, an welchem mich eine ungewöhnlich harte Magen-Darm-Grippe erwischt. Aber zum Eintrittstag ins Spital, wo mich eine Operation als Folge einer verkannten Diskushernie erwartet, bin ich einigermassen wieder auf dem Damm.

\section{Bloss nichts Neues, nicht auch noch Wanzen.}

Dass ich während des ganzen Jahres 2017 immer wieder von Übelkeit und einer ungewöhnlichen Mattigkeit heimgesucht werde, stecke ich weg. Es gibt ja auch gute Tage, manchmal sogar ein oder zwei gute Wochen. Eine Magenspiegelung bei meinem Gastroenterologen lässt ihn eine Laktoseintoleranz vermuten. Bloss nichts Neues, nicht auch noch Wanzen. Es soll doch bei den mir vertrauten und ins Leben integrierten Läusen und Flöhen bleiben.

Dann die Grippe im Dezember. Am fünften Tag beschert mir ein tiefer Blutdruck einen Kollaps, und ab sofort beherrscht mich eine Übelkeit, die mir jegliche Nahrungsaufnahme verunmöglicht. Primperan soll es richten. Im nahe gelegenen Spital wird eine Diagnostik eröffnet, mein Innenleben akribisch untersucht, mit und ohne Kontrastmittel, mit und ohne PropofolKurzschlaf. Aber die Übelkeit bleibt.

"In spätestens zwei Wochen werden Sie wieder gesund sein», so der leitende Arzt. "In die Hand versprochen?»,

\section{Diagnosen}

- Laktoseintoleranz

- Prolongierter viraler Infekt mit Begleithepatitis

- Vermutung: Prolaktinom

- Unklare Schwäche nach prolongiertem grippalem Infekt

- Histaminintoleranz

- EssentiellerTremor

- Nierenstau links und kleine Zyste, Leber etwas heller als normal

- Hiatushernie mit Reflux

- Gicht im Mittelfinger rechts

- Urtikaria

- Mastzellaktivierungssyndrom frage ich. «Ja!» Ich sitze vor meinem Frühstück, meinem Mittagessen, meinem Nachtessen. «Nur ein wenig!», ermuntert mich mein Mann. "Es geht nicht», antworte ich. Mit der 'Diagnose' «Prolongierter viraler Infekt mit Begleithepatitis» und einem Primperan-Rezept werde ich in die Weihnachtstage entlassen. «Ich bin sicher», sage ich bei der Konsultation im Januar, spürend, dass da Hypothesen entstanden sind, die beigezogen werden, wenn objektiv nichts gefunden werden kann, «dass das nichts mit der Seele zu tun hat.» - «Tja!», meint der Arzt, verordnet eine noch gründlichere Blutuntersuchung und gibt mir einen weiteren Sprechstundentermin.

Da sitze ich nun und warte, als er mich lachend in sein Sprechzimmer bittet: "Prolaktinämie! Sie haben also doch recht gehabt. Somatisch das Ganze!» Die fast achtfache Erhöhung des Hormonspiegels hinsichtlich Prolaktin lässt an ein Tumörchen am Hypophysen-Vorderlappen denken. Trotz Zweifel meinerseits - weshalb sollte ein erhöhter Prolaktinwert eine solche Übelkeit verursachen? - liege ich nun in der Röhre und weiss bereits, dass das MRI mit den Worten «ohne Befund» enden wird.

Was bekannt und objektivierbar ist, wurde getan. Meine Recherche ergibt, dass das Primperan den Prolaktinspiegel erhöhen kann, wiewohl kaum in so astronomische Werte wie bei mir. Ich merke, dass der Arzt in seiner Ratlosigkeit wieder an die Seele denkt. Ich setze das Primperan ersatzlos ab. Dafür setze ich mich mit einer Endokrinologin in Verbindung und bekomme innerhalb von drei Tagen einen Termin. Am drittletzten Januartag schreibt mir die Ärztin:

"Leider kann ich Ihnen nicht wesentlich weiterhelfen, glücklicherweise sind die Hormone in Ordnung [...] Ich habe Sie nur so rasch zwischenrein genommen, um sicher eine Nebennieren-Insuffizienz oder Hypophysenproblematik auszuschliessen, und das konnten wir erfreulicherweise. Die Notfalltermine diese Woche werden nun von anderen dringenden Patienten gebraucht."

Soll ich mich nun freuen? Oder doch nicht? Wäre eine klare Diagnose nicht einfacher als die Ungewissheit? Habe ich nun doch auch noch Wanzen? Aber wie heissen die? Bislang habe ich Doktor Google und jene 
Patienten, die ihn konsultieren, lächelnd abgetan. Jetzt sitze ich vor dem Computer und gebe Worte ein: «Permanente Übelkeit. Mattigkeit.» Klick! Ich warte. Eine Reihe von Vorschlägen taucht auf. Wo beginnen? Mastozytose? Nein, das passt nicht. Mastzellaktivierungssyndrom? Hierzu gibt es mehrere Möglichkeiten. Immer tiefer gerate ich in diese Recherche, und immer giftiger blicken mich die dazugehörigen Symptome an. «Bauchkrämpfe, Übelkeit, Abgeschlagenheit, Juckreiz, tiefer Blutdruck.» Es fällt mir auf, dass ich mich seit Wochen ständig überall kratze und mehr und mehr braunrote Punkte meine Haut zieren. Ich schreibe das Wort auf einen Zettel, den ich bei der nächsten Konsultation dem Arzt unterbreiten will.

\section{Wäre eine klare Diagnose nicht einfacher als die Ungewissheit?}

"Mastzellaktivierungssyndrom? Nein, das glaube ich nicht", meint der Arzt, "ich kann mich natürlich irren, aber ich glaube es nicht.» Wieder bin ich allein, mir ist seit Wochen hundeübel. Wenn ich morgens aufstehe, zwinge ich mich, etwas zu essen, hänge mich in einen bequemen Sessel und schaue zum Fenster hinaus, bis der Terminkalender mich in die Praxis drängt. Zwischen der Behandlung von zwei Patienten lege ich mich auf meine Matte, die mir sonst für die Arbeit mit Kindern und für Patienten dient, welche eine Entspannungsübung benötigen. «Ob man mir im Unispital helfen könnte?», frage ich den Arzt und ergänze: «Lange halte ich diese Übelkeit nicht mehr aus.»

"Du hast abgenommen", sagen Nachbarinnen, und "Sind Sie krank?», fragen einzelne Patientinnen. «Neinnein, alles in Ordnung», lüge ich. Aber Doktor Google hat mir einen Hinweis gegeben, den ich irgendwo hintragen muss. Aber wohin? Gibt es irgendwo einen Arzt oder eine Ärztin, der oder die es aushält, keinen Namen für mein Leiden zu kennen? Ein Mail ins Unispital soll mir helfen. Ich lande auf der Abteilung Hämatologie und wende mich an einen der leitenden Ärzte:

\section{Februar 2018, 9.06 Uhr}

«Sehr geehrter Herr Doktor B.

Ich wende mich an Sie in einer Notsituation. Kurz geschildert:

- Seit mehr als zwei Monaten Übelkeit, die nie mehr wegging

- Müdigkeit und Schwächezustände

- Abgeklärt in wohl allen Disziplinen immer ohne Befund

Ich bin sehr ratlos und kann meinen Alltag fast nicht mehr bewältigen. Könnte es sich um ein Mastzellaktivierungssyndrom (MCAS) handeln? [...]

Freundliche Grüsse»
Ich warte. Hie und da schaue ich ins Mail-Böxli. Es schweigt. Ich greife nochmals in die Tasten:

\section{Februar 2018, 16.19 Uhr}

"Sehr geehrter Herr Doktor B.

Mein Arzt, Dr. C. (vom Dings-Spital) wird mich an Sie überweisen und ich bin Ihnen sehr dankbar, wenn ich möglichst bald bei Ihnen einen Termin bekommen darf. Vielen Dank im Voraus und freundliche Grüsse»

\section{Februar 2018, 17.32 Uhr}

«Sehr geehrte Frau B.,

Wir werden Sie in meine Sprechstunde aufbieten lassen. Dies wird aber frühestens in 4 Wochen möglich sein. Ist das OKfür Sie? [...]

Grüsse, St. B.»

\section{Februar 2018, 18.43 Uhr}

«Sehr geehrter Herr Doktor B.

Vier Wochen, das macht mir sehr Angst, [...] ich kann fast nicht arbeiten, [...] weiss nicht, wie ich diese Zeit überstehen soll. Ich kann fast keiner Verpflichtung nachkommen (bin praktizierende Psychotherapeutin und muss als Präsidentin des Blinden- und Sehbehindertenverbandes von $\mathrm{ZH} / \mathrm{SH}$ Sitzungen leiten), aber ich schaffe kaum den Haushalt [...] Ich hoffe so sehr auf Ihre Hilfe [...] Wenn immer möglich wäre ich Ihnen unglaublich dankbar, wenn ich früher kommen dürfte. In der Hoffnung, von Ihnen zu hören, grüsse ich Sie freundlich.»

Das Telefon klingelt. Doktor B. am Apparat. Acht Tage statt vier Wochen. Das muss ich aushalten. Er erhört meine Not, ich kann auf den 14. Februar in seine Sprechstunde. Mein Mann begleitet mich. Wir irren durch die Korridore des Unispitals, suchen die richtige Etage.

Wir reparieren mehr Herzen pro Jahr, als Casanova in seinem ganzen Leben gebrochen hat.

So steht es auf der Wand gegenüber des Aufzugs. Na also, dann wird man ja wohl auch mir helfen können. Doktor B. hat eine Verdachtsdiagnose: Histaminintoleranz. Verordnet Antihistaminika.

Knappe zwei Wochen später kann ich ihm berichten, dass die Übelkeit unter den Medikamenten etwas besserte, muss aber ergänzen, dass ich das eine nicht ertrage. Die Müdigkeit als Nebenwirkung macht mir den Alltag auf eine andere Art schwierig. Des Zitterns meiner Hände wegen rät er mir, bei meiner Neurologin vorstellig zu werden.

Am 15. März finde ich mich bei Frau Doktor V. ein. Sie kennt mich seit Jahren. Der Läuse wegen. Jetzt weiss auch sie eine neue Diagnose: essentieller Tremor, rät zu weiteren internistischen Abklärungen.

Erneut zu Doktor B. Wieder prangen Buchstaben von einer Wand, gleich einem Menetekel. 
Wir forschen jetzt, damit Sie später alt werden.

Aber ich bin doch schon 68-jährig. Das ist doch schon ein wenig alt. Später noch älter? Mit dieser Befindlichkeit? Das kann ich mir nicht vorstellen. Doktor B. verschreibt ein anderes Antihistaminikum. Es folgen drei Traumtage. So habe ich mich mal gefühlt. Dann die Verwirrung. Wo bin ich? Was ist Sache? Mein Mann liest den Beipackzettel. Unter den Nebenwirkungen steht, dass das Mittel auf das Zentrale Nervensystem einwirkt und oft mit Verwirrung einhergeht. Was jetzt? Verwirrung oder Übelkeit.

Frühlingsbeginn. Wir landen wieder im Unispital. Doktor B. nimmt Kontakt auf mit dem Allergologen Professor Sch., der mich und meine Allergien seit Jahren kennt. Verordnet Ernährungsberatung, nochmals eine Magenspiegelung und ein Ultraschall. Die erneute Abklärung führt mich im Unispital auf neue Wege. Ein neues Menetekel.

\section{Wir bieten dem Krebs die Stirn.}

Okay, dann werden sie auch meinem Leiden die Stirn bieten. Zumindest bekomme ich eine neue Diagnose: Der Magen ausser einer kleinen Hiatushernie mit Reflux so weit in Ordnung, aber links sind ein Nierenstau und eine Zyste auszumachen. Die sollen weiterverfolgt werden. Nein danke, lieber nicht. Ich will die Übelkeit loswerden. Sonst nichts.

\section{Gibt es irgendwo einen Arzt oder eine Ärztin, der oder die es aushält, keinen Namen für mein Leiden zu kennen?}

Auf der Allergiestation empfängt mich eine Assistenzärztin. Sie schaut sich die Entzündung auf der rechten Hand an. "Gicht im Mittelfinger», sagt sie. «Nein!», antworte ich, «ich kann das Gelenk ja problemlos bewegen.» Jetzt werden im Grosscheck neue Allergien festgestellt. Ein Apérohäppchen mit Fisch drin bescherte mir kürzlich innerhalb einer halben Stunde einen Kollaps. Gut, dass ich Fische ohnehin nicht mag. Der Dorsch wäre jetzt neu ein Feind auf der Liste der verbotenen Nahrungsmittel. Ich zeige Professor Sch. die immer wiederkehrenden gelb-blauen Flecken auf meiner Haut. "Urtikaria», sagt er. Seine Diagnose jetzt ausserdem: Verdacht auf ein Mastzellaktivierungssyndrom. Ja, hallo, Herr Doktor Google, waren wir nicht auch schon mal so weit?

$\mathrm{Ab}$ nun bekomme ich monatlich eine Injektion im Marktwert von 1200 Franken. Irgendwo im Immunsystem soll diese Gutes leisten. "Jetzt bin ich wieder 1200 Franken mehr wert», sage ich zur Pflegefachfrau, die soeben die Injektion vorgenommen hat. "Ach ja», ant-

\section{Ja, hallo, Herr Doktor Google, waren wir} nicht auch schon mal so weit?

wortet sie, "das baut sich aber schnell ab." Stimmt, denke ich. So viel Wert haben wir Menschen gar nicht. Ich begebe mich ins Haupthaus. Verirre mich erneut. Auf welcher Etage bin ich jetzt? Ein neues Menetekel an der Wand.

\section{Mein Schlaganfall hielt sich nicht an Öffnungszeiten.}

Ja, ein Glück, dass hier stets offen ist, auch, dass ich keinen Schlaganfall habe, denke ich und verlasse das Spital. Eine Woche Cortison beschert mir eine Woche weniger Mattigkeit.

Immer wieder Termine bei Doktor B. Noch immer lässt er sich nicht beirren, obwohl wir ja nicht sicher wissen, ob die letzte Diagnose, die dank Doktor Google eine meiner ersten war, stimmt. «Versuch - Irrtum", sagt er. Ein neues Medikament soll das Histamin austricksen. Und streng halte ich mich an die Diätmassnahmen.

Es gibt gute und weniger gute Tage, aber das unglaubliche Leiden vor einem Jahr scheint durch die Massnahmen gebessert. Eine Bestätigung der Diagnose? Egal. Ich bleibe dran. Gern gemeinsam mit Doktor B. und Professor Sch., die mich begleiten, auch wenn mein Leiden durch kein MRI, keine Gastroskopie, kein CT und kein Röntgen einen Namen bekommen kann.

\section{Im USZ änderten sie schon Profile, bevor ich meins auf Social Media hatte.}

$\mathrm{Na}$ dann, weiter $\mathrm{zu}$. Ich weiss heute nach eineinhalb Jahren, dass ich mit gewissen Symptomen leben lernen muss, und bin Doktor Google, Doktor B. und Professor Sch. dankbar für ihre Unterstützung auf diesem Weg.
Erica Brühlmann-Jecklin

ebj[at]hispeed.ch www.ebj.ch 